\title{
Low-power system for the acquisition of the respiratory signal of neonates using diaphragmatic electromyography
}

This article was published in the following Dove Press journal:

Medical Devices: Evidence and Research

22 February 2017

Number of times this article has been viewed

\section{Róbinson Torres ${ }^{1,2}$ \\ Sergio López-Isaza ${ }^{1,2}$ \\ Elisa Mejía-Mejía ${ }^{1,2}$ \\ Viviana Paniagua ${ }^{1,2}$ \\ Víctor González ${ }^{3}$}

'Biomedical Engineering Department, Universidad EIA, Envigado, ${ }^{2 B i o m e d i c a l}$ Engineering Department, Universidad CES, ${ }^{3}$ Neonathology Department, Hospital General de Medellín Luz Castro de Gutiérrez, Medellín, Antioquia, Colombia
Correspondence: Róbinson Torres Biomedical Engineering Department, Universidad EIA, Calle 25 Sur 42-73, Envigado, Antioquia 055420, Colombia Tel +57 43549090 ext 1316 Email robinson.torres@eia.edu.co
Introduction: An apnea episode is defined as the cessation of breathing for $\geq 15$ seconds or as any suspension of breathing accompanied by hypoxia and bradycardia. Obtaining information about the respiratory system in a neonate can be accomplished using electromyography signals from the diaphragm muscle.

Objective: The purpose of this paper is to illustrate a method by which the respiratory and electrocardiographic signals from neonates can be obtained using diaphragmatic electromyography. Materials and methods: The system was developed using single-supply, micropower components, which deliver a low-power consumption system appropriate for the development of portable devices. The stages of the system were tested in both adult and neonate patients.

Results: The system delivers signals as those expected in both patients and allows the acquisition of respiratory signals directly from the diaphragmatic electromyography.

Conclusion: This low-power system may present a good alternative for monitoring the cardiac and respiratory activity in newborn babies, both in the hospital and at home.

Significance: The system delivers good signals but needs to be validated for its use in neonates. It is being used in the Neonatal Intensive Care Unit of the Hospital General de Medellín Luz Castro de Gutiérrez.

Keywords: apnea, diaphragm, electromyography, neonates, respiratory signal

\section{Introduction}

The immaturity of the body systems of neonates, specifically the respiratory control systems, induces the respiration patterns in neonates to vary between eupnea, apnea, sigh, and an irregular breathing called periodic breathing. ${ }^{1}$ These respiratory patterns do not always require any treatment, although they lead to the presence of aperiodic patterns in the breathing of infants and neonates. ${ }^{2}$ However, sometimes these patterns require special attention and are considered as clinical events, as when an apnea episode occurs. An apnea episode is defined as the cessation of breathing that lasts $>20$ seconds or $>10$ seconds and is accompanied by oxygen desaturation or bradycardia. ${ }^{3}$ These episodes have been found in $>50 \%$ of preterm infants, mainly in those whose weight at the time of birth is $<1000$ g. ${ }^{2,3}$ As stated by Belal et al, ${ }^{4}$ recurrent apnea episodes are related to retinopathy of prematurity, necrotizing enterocolitis, and periventricular leukomalacia.

In preterm babies, the apnea is classified as central, obstructive, or mixed. ${ }^{3}$ According to Hansen and Corbet, $40 \%$ of the episodes correspond to central apnea, whereas $10 \%$ are obstructive apnea and the remaining $50 \%$ are mixed. ${ }^{5}$ Differentiation between these episodes is important to understand the physiopathology of the apnea in neonates, in order to improve its prognosis and treatment. 
Neuman $^{6}$ assures that the respiratory activity is the main electrical action that is monitored in neonatology, especially for the identification of the apnea episodes. For this monitoring, several methods have been established, both direct (the sensor element is coupled to the airway of the patient and measures the properties of the air that is transported in and out of the lungs) and indirect (the sensor measures variables related to the movement of the air, but not the properties of the air); ${ }^{6}$ one of the most used techniques for monitoring the respiratory activity in neonates is the thoracic impedance measurement, and some vital signs monitors use algorithms to detect the apnea episodes using this variable. ${ }^{3}$ Nonetheless, as Beck et al ${ }^{1}$ established, this can be considered as a mechanical signal and "may not reflect the true neural respiratory center output."

A vital signs monitor specialized for the detection of neonate apnea events was developed by Ochoa et al. ${ }^{2}$ This system was based on the acquisition of the respiratory signal of neonates from the analog processing of the electromyography signal of the diaphragm muscle (EMGdi) obtained using surface electrodes. In general, the electromyography signal (EMG) delivers information related to the neuromuscular activity. ${ }^{7}$ Measurement of the electrical activity of the diaphragm is a direct way to monitor the respiratory signal and the neural signals for breathing. ${ }^{1,2}$ This device uses EMGdi (the diaphragm is the main muscle for breathing in neonates) and electrocardiography (ECG) in order to detect the apnea episodes, arguing that a noninvasive device for the measurement of the EMGdi that estimates the lung function can increase the possibilities of diagnosis and retrieve an objective measurement to detect the apnea episodes. Moreover, the classification between central apnea and obstructive apnea can be done by measuring the electrical activity of the diaphragm due to the direct relation between the EMGdi and the central nervous system. Besides, EMGdi and ECG may be used as indirect variables to estimate the obstruction of the airway. ${ }^{2}$

Monitoring the respiratory function using noninvasive EMGdi seems attractive. However, the main issue with this technique is the interference generated by the ECG that appears as noise due to the placement of the electrodes. ${ }^{8}$ This interference is greater than the amplitude of the signal and belongs to the frequency band of the EMGdi, making it hard to process an EMGdi and acquire a reliable respiratory signal unless the ECG signal (mostly the QRS complex) that appears as noise is eliminated or reduced. The techniques that have been used to face this problem are the adaptative filters, the analog masking, and the signal clipping. ${ }^{8}$

In this study, the development of an analog system for the elimination of the ECG signal present on the EMGdi, based on the system developed by Ochoa et al, ${ }^{2}$ is presented. The original system was modified using single-supply, low-power consumption electronic components. The singlesupply approach allows the system to be battery powered, ${ }^{9}$ particularly when micropower components are used, and has become very popular due to the portability that can be acquired in the devices using single-supplied circuits powered by a single battery, even though the design of these singlesupply circuits is difficult due to the requirement to account for input references. ${ }^{10}$

The system developed uses single-supply voltage and features low current consumption, making it portable and ideal for coupling with a microcontroller or microprocessor in order to perform real-time apnea detection.

\section{Materials and methods}

The developed system has four main stages that allow the extraction of the respiratory signal and the ECG using only three electrodes and one input channel. Its block diagram is shown in Figure 1.

The first stage of the system consists of an initial amplification of the biomedical signals that are acquired by the electrodes; this is the EMGdi that carries the ECG (Figure 1(a)). The electrodes are positioned on top of the patient's diaphragm: on the breastbone (as a reference) and below the costal margin vertically aligned with the nipples, both left and right (Figure 2). ${ }^{2}$ In this stage, the interference signal is eliminated using a band pass filter with passband of $0.5-400 \mathrm{~Hz}$ (Figure 1(b)). The principal objective of this stage is to raise the signal-to-noise ratio (SNR), while the mixed signal that has both the EMGdi and the ECG (EMG + ECG) is obtained. A high SNR is important in order to protect the EMGdi and ECG signals from electromagnetic and other nondiaphragmatic muscle interference, such as the intercostal muscle activity.

The acquired signal is divided into three different branches using filters with different cutoff frequencies according to the characteristics of the signals to be obtained. The upper branch (Figure 1(c)) consists of a second-order band pass Butterworth filter with cutoff frequencies of 0.5 and $40 \mathrm{~Hz}$; this delivers as a result of the ECG signal.

The middle branch in the block diagram includes a band pass Butterworth filter $(9-20 \mathrm{~Hz})^{11}$ for the acquisition of the QRS complex of the ECG signal, an amplification stage and a level comparator (Figure 1(d-f), respectively). The purpose of this stage is to obtain a pulse signal synchronized to the QRS complex of the ECG signal.

Due to the spectral components of the QRS complex, they are the main interference source in the EMGdi, which has 


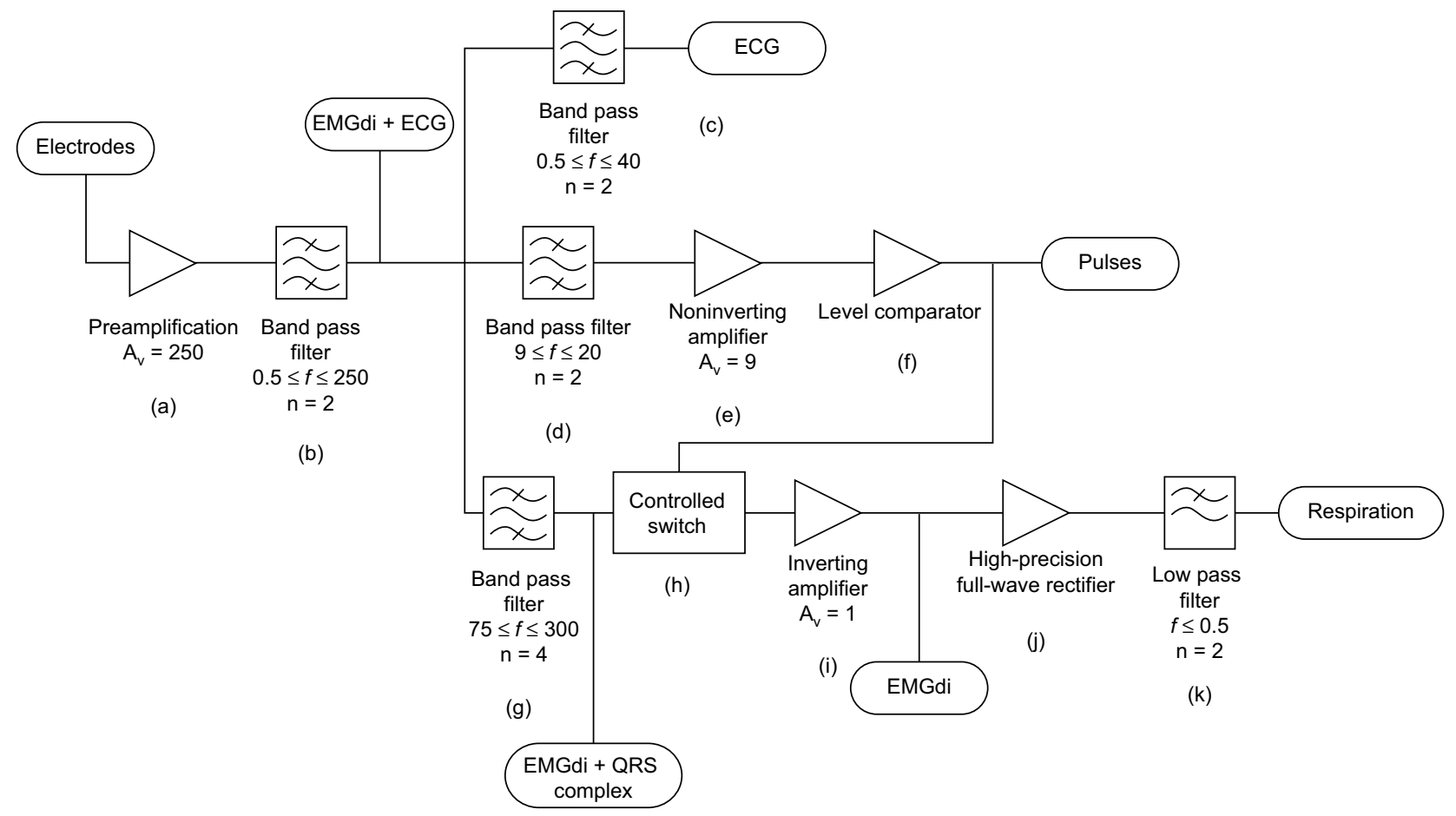

Figure I Block diagram of the system for the acquisition of EMGdi, ECG, and respiratory signals.

Notes: (a) Preamplification stage with a 250 gain. (b) Bandpass filtering stage for the removal of high frequency noise. (c) Bandpass filtering stage for the acquisition of electrocardiography. (d) Bandpass filter for the detection of QRS peaks in ECG. (e) Amplification stage. (f) Level comparator for the detection of pulses. (g) Bandpass filtering for the acquisition of EMG signal. (h) Controlled switch for the deletion of ECG signal merged in EMG signal. (i) Amplification stage. (j) Full-wave rectification for the extraction of respiratory signal.

Abbreviations: ECG, electrocardiography; EMGdi, electromyography signal of the diaphragm muscle.

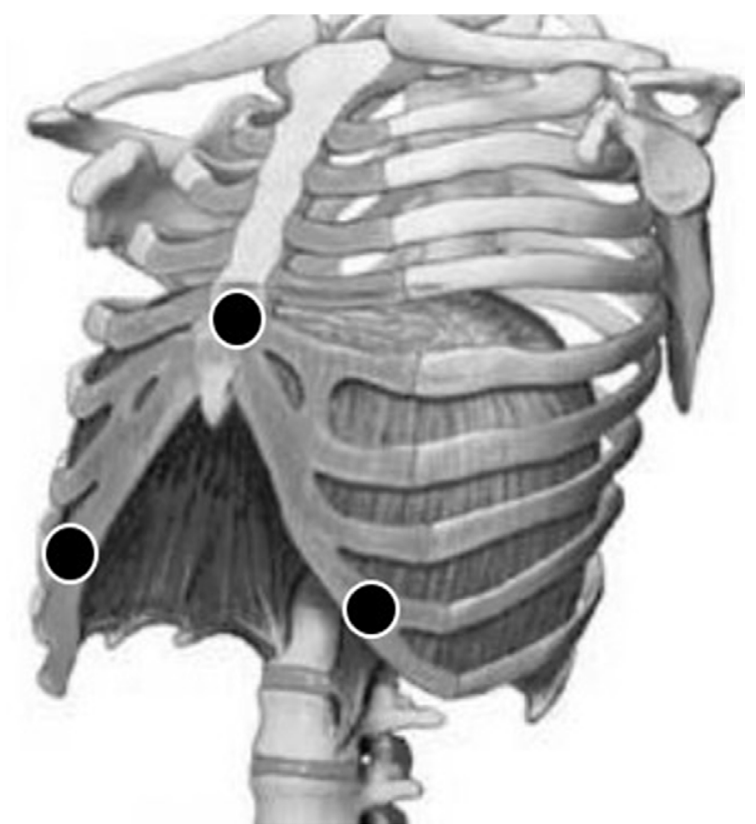

Figure 2 Positioning of the electrodes (black circles) for the acquisition of EMGdi. Abbreviation: EMGdi, electromyography signal of the diaphragm muscle.

frequencies between 70 and $300 \mathrm{~Hz}$. Therefore, an elimination of this component of the ECG signals must be performed in the EMGdi signal. However, this removal cannot be made using conventional filters as the spectral components of both signals overlap. The pulse signal acquired in the previous branch is essential to accomplish the removal of the QRS complexes in the EMGdi.

The lower branch corresponds to the obtainment of the EMGdi and the extraction of the respiratory signal. First, it is necessary to apply a band pass Butterworth filter (cutoff frequencies of 75 and $300 \mathrm{~Hz}$ ) to the EMG + ECG signal (Figure 1(g)), which allows removal of most of the interference in the EMGdi signal, except for that generated by the QRS complex. The signal obtained is denoted as EMG $+\mathrm{QRS}$. The interference generated by the complexes is deleted using a switch controlled by the pulse signal (Figure 1(h)). The signal goes through whenever the pulse signal is in high level $(5 \mathrm{~V})$, but when it is in low level the switch delivers a zero to its output, deleting the QRS complex from the input signal. Once the QRS is deleted, the EMGdi signal is conditioned to improve its SNR (Figure 1(i)) obtaining a resulting signal called EMG.

Then, the EMG signal goes through a high-precision full-wave rectifier (Figure 1(j)) that allows the obtainment of the EMGdi power, which is then low pass filtered with a small cutoff frequency according to the expected respiratory frequency of the neonates (Figure 1(k)). Therefore, an envelope signal (ie, respiratory signal) is obtained from the diaphragmatic activity. 
The developed system was designed, constructed, and finally assembled in a compartment along with a pulse oximetry system and an AC-to-DC converter (MWA100018A12A, Inventus Power, Woodridge, IL, USA). All these items were selected and designed taking into account the patients' safety, medical regulations, and the electromagnetic compatibility within the system and other medical equipment. The systems were connected to a data acquisition system (NI USB-6281; National Instruments, Austin, TX, USA), which digitizes and sends the signals to a computer using a virtual instrument (VI) developed in LabView (National Instruments). The signals were visualized and stored in the computer for further processing and analysis. This system was implemented in the Hospital General de Medellín Luz Castro de Gutiérrez (HGM) in order to acquire data from the neonate patients. Written informed consent was obtained from all adult subjects and from the legal representatives of all neonate subjects. The study was approved by the Universidad CES ethics committee.

Figure 3 illustrates the experimental setup in the HGM. This was built in the neonates intensive care unit (NICU) and controlled all the time by at least one of the researchers.

\section{Results}

Figure 4 shows the signals obtained from an adult and healthy patient, and Figure 5 shows the signals obtained from a neonate patient hospitalized in the HGM.

\section{Discussion}

As can be seen in Figure 4, the stages mentioned in Figure 1 result in the expected signals. The raw EMGdi signal (Figure 4A) has a notorious interference due to the ECG signal, mainly the QRS complexes. Meanwhile, although other muscles interference are possibly found on the EMGdi signal as the surface electromyography technique cannot differentiate the muscle branches that are being activated,

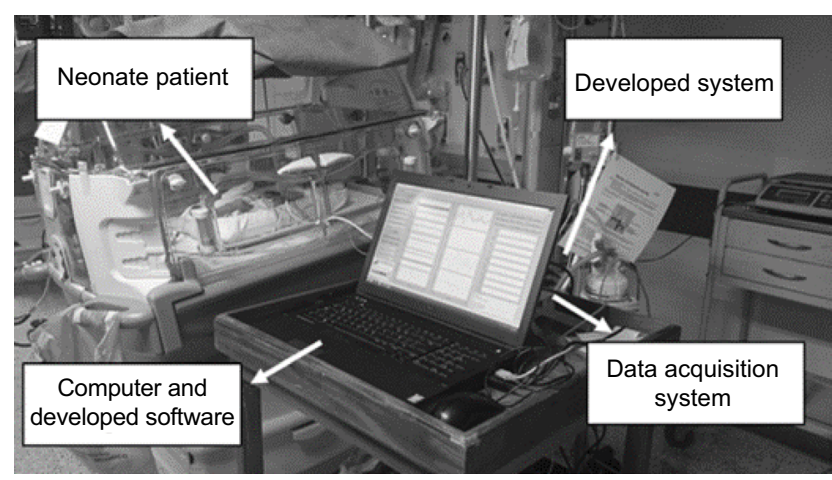

Figure 3 Experimental setup in the neonates' intensive care unit of the Hospital General de Medellín Luz Castro de Gutiérrez (Medellín, Antioquia, Colombia).
A

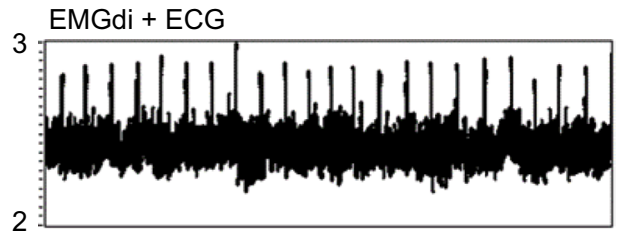

B

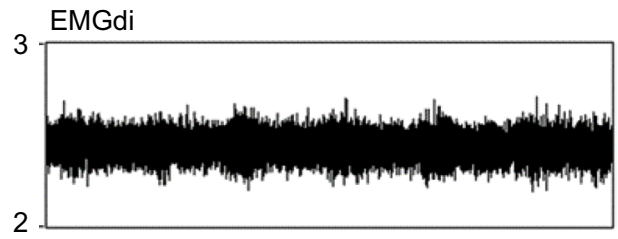

C

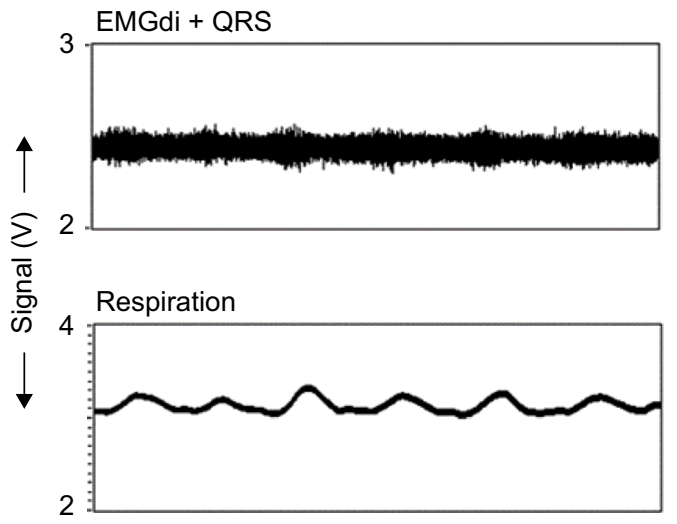

E

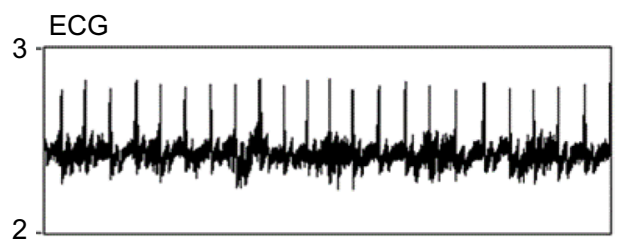

$\mathbf{F}$

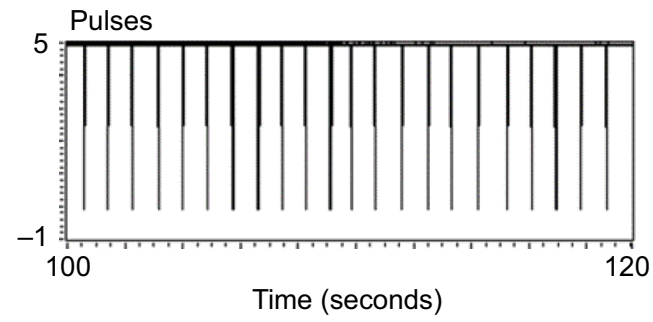

Figure 4 Signals obtained from an adult patient in a 20 -second period. Notes: (A) Signal obtained by the electrodes after the preamplification stage (Figure I (a) and (b)); (B) EMGdi after the removal of the whole ECG signal, including the QRS complexes (Figure I (h) and (i)); (C) EMGdi after the ECG elimination filter (Figure I (g)); (D) respiratory signal obtained from the EMGdi and its processing (Figure I (j) and (k)); (E) ECG signal extracted from the EMGdi (Figure I (a)); and (F) pulse signal used for the elimination of the QRS complex from the EMGdi (Figure I (d)-(f)).

Abbreviations: ECG, electrocardiography; EMGdi, electromyography signal of the diaphragm muscle.

this interference is not significant for the acquisition of the respiratory signal from the diaphragm, due to the location of the electrodes ${ }^{8,12}$ and the nature of the respiratory process in humans, in which the maximum effort during respiration is done by the diaphragm. ${ }^{13}$

The removal of ECG-generated interference is essential in order to obtain a respiratory signal as clean as possible. 
A

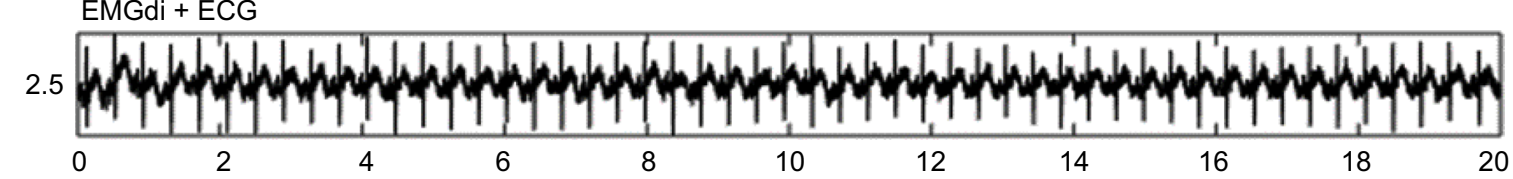

B

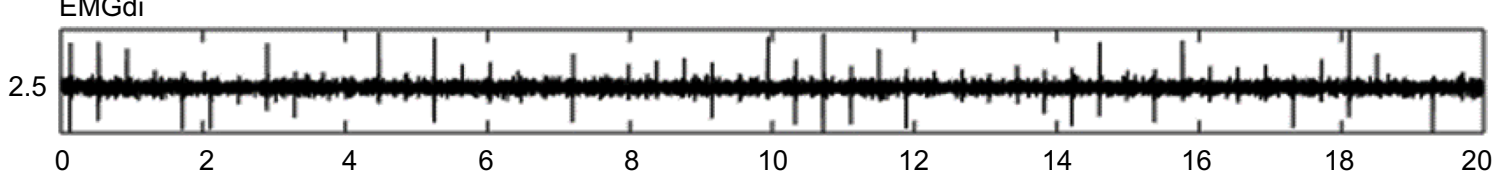

C

$\mathrm{EMGdi}+\mathrm{QRS}$

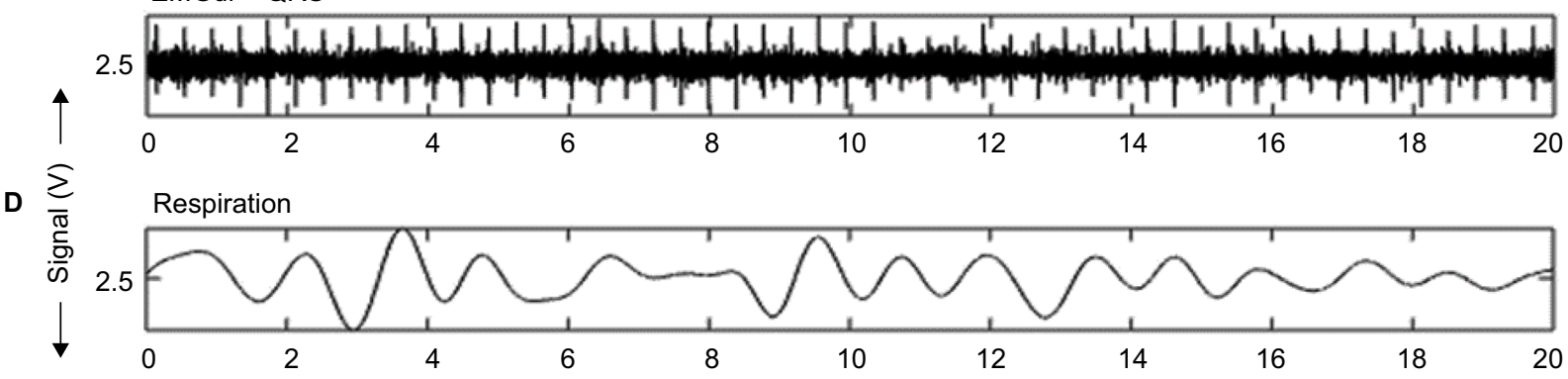

$\mathbf{E}$

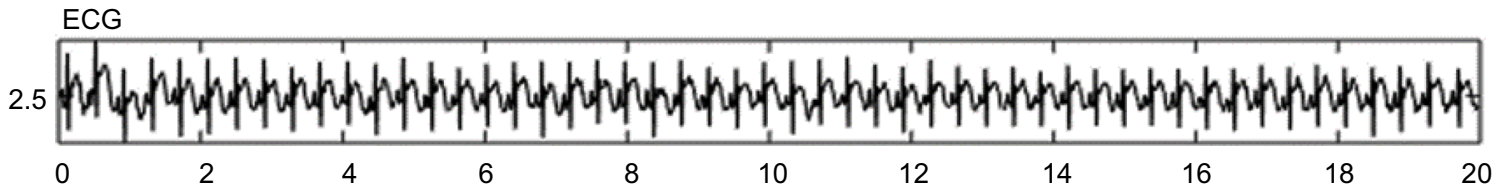

$\mathbf{F}$

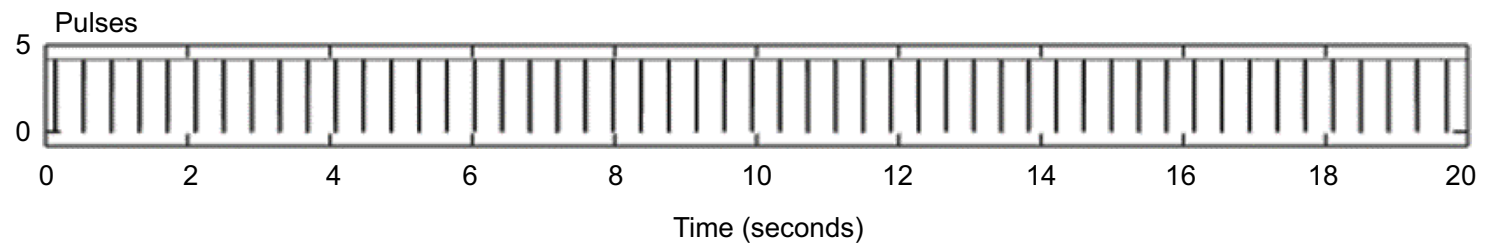

Figure 5 Signals obtained from a neonate patient in the neonatal intensive care unit of the Hospital General de Medellín Luz Castro de Gutiérrez (Medellín, Antioquia, Colombia) during a period of 20 seconds.

Notes: (A) Signal obtained by the electrodes after the pre-amplification stage; (B) EMGdi after the complete elimination of the ECG; (C) EMGdi after the filter for the attenuation of the ECG; (D) respiratory signal obtained using the EMGdi; (E) ECG signal extracted from the EMGdi; and (F) pulse signal used to remove the QRS complex from the EMGdi.

Abbreviations: ECG, electrocardiography; EMGdi, electromyography signal of the diaphragm muscle.

The designed filters and the pulse-controlled switch make it possible to acquire a reliable respiratory signal, deleting most of the ECG components present in the EMGdi.

On the other hand, the ECG signal (Figure 4E) after the extraction of the EMGdi is cleaner, although it still carries some noise from the EMGdi signal when its amplitude increases, ie, the patient breathes. As the QRS complexes are well defined, they are ideal for the extraction of the pulse signal (Figure 4F).

An important factor that must be considered is the synchronization of the used filters so that the delays present in the different signals are similar enough. This is to assure that the fraction of signal eliminated by the switch corresponds to the QRS peaks present as noise in the EMGdi and that no important components of this signal are eliminated. In this design, the synchronization is accomplished using different order filters: the ECG signal filter is order 2, whereas the EMGdi signal filter is order 4 . The ECG signal has components mainly between 0 and $40 \mathrm{~Hz}$. At $40 \mathrm{~Hz}$, the group delay is $\sim 4664 \mu \mathrm{s}$. On the other hand, for the EMGdi signal at $40 \mathrm{~Hz}$, the group delay is $\sim 8000 \mu$ s. Once filtered, the ECG signal (now consisting only on the QRS complexes) is amplified and then compared to a threshold. These last two stages (the amplification and the level comparator) have a delay as well, making the gap between both signal delays very short.

As expected the signals from both adult and neonate patients are different, having more components in the neonate than in the adult patient both in the ECG and in the EMGdi. This is due to the heart rate and the respiratory frequency of the neonates.

The respiratory signal in the EMG signal from the neonate (Figure 5C) is not as well defined as in the adult patient (Figure 4B), and the extracted respiration signal (Figure 5D) 
is harder to obtain, making it very important to perform a precise digital processing for further analysis. This increased noise in the EMGdi signal from neonates can be due to the environment where the system is located, because in the NICU several devices operate at the same time and can generate interference in the signals. Hence, a well-designed isolation system must be taken into account in order to enhance the quality of signals from neonates.

Clinical trials are being executed in order to obtain validation of the technique, both for the acquisition of the respiratory and the ECG signal, comparing these with gold-standard techniques, and for checking if the respiratory signal is indeed a good marker for the early detection and differentiation of central and obstructive apnea.

\section{Conclusion}

The system designed and constructed delivers a response as expected and allows the consecution of respiratory signals directly from the EMGdi. From the original signal (EMGdi), it is also possible to acquire a good-quality ECG. Therefore, it is possible to consider the development of vital signs monitoring systems for neonates, which require fewer electrodes and cables, improving the handling of the patients and their comfort in the clinical facilities.

Moreover, the system is single supplied and delivers signals centered at $2.5 \mathrm{~V}$. This is crucial to integrate it with microcontroller or microprocessor systems and opens the door for developing simple and low-cost modular solutions.

In further works, the utility of the EMGdi to determine the apnea episodes will be verified: the relationship between the apnea episodes and some characteristics of the EMGdi, the ECG, and of the pulse oximetry signals, will be analyzed so that an early detection of these episodes can be achieved.

\section{Acknowledgments}

The authors thank the Colombian Royalties National System and Medellín's business and innovation center, Ruta N, for the management and donation of the resources, which fund this research. This study was sponsored by the Colombian Royalties National system and Medellín's business and innovation Center, Ruta N.

\section{Disclosure}

The authors report no conflicts of interest in this work.

\section{References}

1. Beck J, Reilly M, Grasselli G, et al. Characterization of neural breathing pattern in spontaneously breathing preterm infants. Pediatr Res. 2011; 70(6):607-613.

2. Ochoa JM, Osorio JS, Torres R, McLeod CN. Development of an apnea detector for neonates using diaphragmatic surface electromyography. In: Proceedings of the 31st Annual International Conference of the IEEE EMB; September 3-6, 2009; Minneapolis, MN.

3. Lee H, Rusin CG, Lake DE, et al. A new algorithm for detecting central apnea in neonates. Physiol Meas. 2012;33(1):1-17.

4. Belal SY, Emmerson AJ, Beatty PC. Automatic detection of apnoea of prematurity. Physiol Meas. 2011;32(5):523-542.

5. Hansen TN, Corbet A. Control of breathing. In: Taeusch HW, Ballard RA, Gleason CA, editors. Avery's Diseases of the Newborn. 8th ed. Philadelphia, PA: Elsevier; 2005:616-633.

6. Neuman MR. Neonatal monitoring. In: Webster JG, editor. Encyclopedia of Medical Devices and Instrumentation. 2nd ed. Hoboken, NJ: WileyInterscience; 2006:11-31.

7. Khezri M, Jahed M. Real-time intelligent pattern recognition algorithm for surface EMG signals. Biomed Eng Online. 2007;6:45. Available at: https://biomedical-engineering-online.biomedcentral.com/ articles/10.1186/1475-925X-6-45. Accessed February 28, 2015.

8. Pang P, Pepper M, Ducker D. Monitoring respiratory activity in neonates using diaphragmatic electromyography. Med Biol Eng Comput. 1995;33:385-390.

9. Kitchen C. Biasing and Decoupling Op Amps in Single Supply Applications. AN-581 [application note]; Analog Devices; 2002. Available from: http://www.analog.com/media/en/technical-documentation/ application-notes/AN-581.pdf. Accessed April 30, 2015.

10. Macini R. Single-supply op amp design. Amplifiers: Op Amps [application note]; Texas Instruments Incorporated; 2005. Available from: http:// www.ti.com/lit/an/slyt189/slyt189.pdf. Accessed April 30, 2015.

11. Elgendi M, Jonkman M, De Boer F. Frequency bands effects on QRS detection. In: Proceedings of the Third International Conference on Bio-inspired Systems and Signal Processing, Valencia, Spain, 20-23 January 2010. Berlin: Springer-Verlag; 2010.

12. Maarsingh EJW. The Electrical Activity of Respiratory Muscles: An Indirect Measure to Estimate Airway Reactivity in Children with Recurrent Wheezing [dissertation]. Amsterdam: University Hospital Amsterdam; 2004.

13. Sheffy J. Recording of the Diaphragm Activity during Anaesthesia [dissertation]. Oxford, UK: University of Oxford; 1994.
Medical Devices: Evidence and Research

Publish your work in this journal

Medical Devices: Evidence and Research is an international, peerreviewed, open access journal that focuses on the evidence, technology, research, and expert opinion supporting the use and application of medical devices in the diagnosis, monitoring, treatment and management of clinical conditions and physiological processes. The identification of novel devices and optimal use of existing devices which will lead to improved clinical outcomes and more effective patient management and safety is a key feature. The manuscript management system is completely online and includes a quick and fair peer-review system. Visit http://www. dovepress.com/testimonials.php to read real quotes from authors. 